\title{
SUICIDE AND SUICIDE ATTEMPT DESCRIPTORS BY MULTIMETHOD APPROACH
}

\author{
Bojan Zalar ${ }^{1}$, Blanka Kores Plesničar ${ }^{1}$, Ina Zalar ${ }^{2}$ \& Matej Mertik \\ ${ }^{1}$ University Psychiatric Clinic Ljubljana, Ljubljana, Slovenia \\ ${ }^{2}$ Faculty of Medicine, Ljubljana, Slovenia \\ ${ }^{3}$ Alma Mater Europea, Maribor, Slovenia
}

received: 10.5.2018;

revised: 7.8.2018;

accepted: 21.8 .2018

\section{SUMMARY}

Background: Suicide is a complex action of suicidal methods and peripheral factors with seemingly threatening components representing actual cause for the suicidal actions. It is especially those, apparently unimportant factors that represent a crucial milestone in the network of all the other, personal, cultural, genetic and biochemical factors, forming the method of action consequently deciding between life and death.

Subjects and methods: Based on the Register of Suicides in the Republic of Slovenia kept by the University Psychiatric Clinic Ljubljana, we used a combination of attributes varying within a variable and between variables. Due to limited application of standard statistical methods and analyses in such cases, we used the Machine learning method, Multimethod hybrid approach, which allows combining of different approaches to machine learning (decision trees, genetic algorithms and supplementary vectors). The research included 56712 persons attempting suicide and 21913 persons committing suicide. We chose a form of a suicide action with both possible results: attempted suicide and suicide.

Results: Based on the analysis of machine learning, we defined attributes of the action regarding their lethal effect: attempted suicide and suicide commitment. The suicide register kept for the last 40 years shows hanging as the most commonly used suicidal method, used by men with the purpose of causing suicidal death rather than a suicidal attempt. On the other hand, use of medicaments is linked to the suicidal attempt and mostly used by females.

Conclusions: All methods of suicidal actions cannot predict suicidal death, thus we examined different methods of suicide to most accurately predict the link between the method and its effect in terms of suicide attempt or suicide. The Machine learning method confirmed the attributes of suicide methods in connection with their different outcomes. This analytical method is useful in processing large databases since it enables one variable's intensity to affect other variables in terms of result and meaning. The identification of the most decisive risk factors for suicidal behaviour can serve as basis for planning an effective prevention strategies, timely identification and adequate proffessional help to the high risk persons.

Key words: suicide commitment - suicide attempt - multimethod

$$
* * * * *
$$

\section{INTRODUCTION}

Worldwide, almost 800000 people die by suicide every year (WHO 2018). Suicide is the second leading cause of death among 15-29 year-olds and the rates of suicide among young people are so far still increasing (WHO 2017). Rates of suicide vary greatly between countries, the greatest burdens being in the developing countries. Although suicide rates in elderly people have fallen in many countries, those in young people have risen. Rates also vary with ethnic origin, employment status and occupation (Hawton \& Heeringen 2009). Hanging, firearms and pesticide poisoning are among the most common methods of suicide globally (WHO 2014). In most countries, the risk of nonfatal suicidal behaviour is higher among young people, women, unmarried and socially disadvantaged people (Hawton \& Heeringen 2009, Hawton et al. 2012). In general, suicide rates are higher for males whereas suicide attempts tend to be higher for females. Even though women tend to choose less lethal methods and men choose high-risk methods it was reported that within each method (with the exception of drowning) case fatalities were higher for men than for women (Cibis et al. 2012). Non-fatal suicidal behaviours are more common than suicides and are believed to occur at least 10 times more frequently than fatal suicides (Nock et al. 2008). Additionally, suicide attempts unlike completed suicides are usually underrepresented because they are not that obvious and not always reported.

Methods used in suicide differ according to their lethality. Two important factors that determine the lethality of the method are time span between the initiation of a suicidal act and expected death (quick methods are more lethal, since they reduce the possibility of intervention and seeking help or possibility to change one's mind), and availability of medical aid (McIntosh 1992).

Both, worldwide and in Europe, the predominant choice of suicidal method for men and women is hanging (Ajdacic-Gross et al. 2008, Värnik et al. 2007). Among European men, hanging was followed by death by firearms and poisoning by drugs respectively. For women on the other hand the second leading cause is 
poisoning by drugs, followed by jumping from high place (Värnik et al. 2007). It may not be surprising that hanging is the most common suicide method in most countries, since it is largely available (Lester 1990). However, suicidal behaviour and the preferred suicide method vary between countries and these differences appear to be more significant than differences between genders. Meaning that if a suicide method is particularly popular in men, this will also be the case in women, and contrariwise (Ajdacic-Gross et al. 2008).

Situation in Slovenia is similar to other countries around the world. Slovenia has the prevalence of suicidality among men and elderly people, while suicide attempts are more common among women and younger people. Suicide is extremely problematic in Slovenia and is at the very top of the world regarding suicidality (Milčinski 2016).

The high shares of hanging for both males and females in Slovenia, are comparable with Hungary and Estonia (Värnik et al. 2007). This similarity was reasoned to be due to certain common features, very high alcohol consumption, high rates of alcohol dependence and rapid socio-political economic changes in integrating into the European Union (Värnik et al. 2007).

\section{Statistic versa machine learning knowledge accumulation}

Many facts were discovered by statistical analyses of the database that were later used by treatment and studies. Statistics, science and practise enables development of human knowledge using empirical data. It is tightly connected with the probability theory in which randomness and uncertainty lead. In statistics, human knowledge is described mathematically and attempts to learn from the observed experiment. This requires to plan the observations as an experiment, summarize a collection of observations to feature their commonality by suppressing details, so-called descriptive statistics, and reach consensus about the observations.

Although a lot of research with statistical approach was made on suicidality, there was not so many done in the recent fields of computer science like machine learning and pattern recognition (Bishop 2006). In some forms of descriptive statistics the second and third of these steps became so prominent that at the same time with the development of computer science, especially the areas of machine learning and artificial intelligence, new field of research was put in practice. It is in a way the statistics that started knowledge extraction and discovery phenomena, nowadays tightly connected with the term of datamining, based on the scientific fields of machine learning, intelligent systems and pattern recognition.

Machine learning principles are gaining important advantages in comparison with pure mathematically statistically described knowledge and have attracted a lot of attention lately in particular the question how the learning capacity can be increased and generalized. The ability to present new knowledge easily and understandably to humans, as well as solving the problem how to learn to learn, still present the main objective in machine learning.

Decision trees proved to be valuable tools for description, classification and generalization of data. Elaborating and constructing decision trees from data exists in multiple disciplines such as descriptive statistic and machine learning, decision theory and pattern recognition. Decision trees are a way to represent rules underlying data with hierarchical sequential structures that recursively partition the data (de Ville 2013).

Another approach to knowledge extraction is to imitate cognitive abilities of the human brain. The most common representative of cognitive approach is artificial neural network. This approach has been verified by the nature and has ability to learn from examples. The main difference from the decision trees is in knowledge representation. The neural network stores the knowledge in the weights of connections to the neurons and has very good characteristics for identification of complex patterns in learning data (Gupta 2013).

Genetic algorithms are adaptive heuristic search methods that may be used to solve all kinds of complex search and optimization problems, for which no efficient heuristic method has been developed. They are based on the evolutionary ideas of natural selection and genetic processes of biological organisms. Simulating the principles of natural selection and "survival of the fittest" first laid down by Charles Darwin genetic algorithms are able to evolve solutions to real-world problems (Sivanandam 2008). They are often capable of finding optimal solutions even in the most complex search spaces or at least they offer significant benefits compared to other search and optimization techniques.

The hybrid approaches rest on the assumption that only in the synergetic combination of single models can unleash their full power. Therefore, the logical step is to combine different methods to overcome the disadvantages and limitations of a single method (Ganguly et al. 2016).

\section{SUBJECTS AND METHODS}

The study population included all individuals registered as committed or attempted suicide in the Register of Suicides of the Republic of Slovenia. The data in the Register are being collected for the whole of Slovenia in co-operation between the University Institute of Forensic Medicine, the Emergency Department of University Medical Centre and the University Psychiatric Clinic. The data about suicides and suicide attempts are very reliable, although there is an uncertainty about the exact number of suicidal attempts. We have focused on the basic epidemiological data on suicide distribution by gender, age, presence or 
absence of diagnosis, methods of actions and mode/ type (attempt or commitment). The database represents the suicide register of the Republic of Slovenia from 1976 to 2016. During this period, on average 434 male and 129 female committed suicide annually, 30 suicides and 64 accidents in 1000 deaths. (Statistical Office of the Republic of Slovenia). According to the coding system in the Register of Suicides, the following modes of suicides and attempted suicides were used: using gas, medicaments, alcohol, poisoning, hanging, drowning, being run over, jumping, shooting, stabbing or cutting, combination of modes or unknown. The data were also arranged as a type of suicidal act into suicides and attempted suicide.

Decision trees are an easy to understand and accepted classification technique in knowledge discovery because of their clarification and flexibility in presenting the classification procedure. Amongst the techniques to build decision tree, Iterative Dichotomiser Tree offered by Quinllan and C4.5 (its enhanced edition) are two of the most accepted methods (Jiang 2009). In the process of classical construction of decision trees, the choice of the most appropriate attribute in the individual node of the decision tree depends on the information content of the attribute, which is evaluated based on the heuristic function. The most frequently used heuristic functions for assessing the information content of the attribute are Information gain ratio derived from entropy (ID3), Chisquare, Gini and J-measure. It is important that we do not use a single methodology when experimenting but find the best. Therefore, we used a Multimethod approach that is based on Genetic algorithms when solving the complex optimisation problems. It decides which information content and decision tree algorithm to use for generating the best possible decision tree on the data. Our Multimethod hybrid approach dynamically combines different methods of machine learning in a common decision tree. Moreover, for discovery of the novel relation between attributes (rules in the tree) the approach makes all possible combination of decision rules considering the database.

\section{RESULTS}

The multimethod constructs all possible combinations of decision rules and uses most promised decision attributes for the construction of the decision tree. In such a way, discovery of the novel relation between attributes (rules in the tree) are produced and then evaluated and used by the experts. This approach is now being integrated as a part of the clinical support system for mental health when treating a suicide. Based on the large data collection study, it represents a valuable tool for getting insight to the patterns in diagnosis in the process of prevention for clinical experts. In classification problems, these techniques are more powerful than the multivariate statistical tests (Baca-Garcia et al. 2007).
The root node of Figure 1 (Suicide Commitment) includes 10004 examples in the learning and 5005 in the test set, similarly all other nodes in the trees are marked. Accuracy of the models was optimized within Multimethod approach based on the unseen examples of the test set. The presented model generated 91\% accuracy in prediction of the decision attribute. The results are from the highest rated set of decision trees accuracies.

With persons committing suicide, the analysis included the presence of psychiatric diagnoses, since about half of the researched population had been psychiatrically treated during life. The dominant group was found to have had abused alcohol, which is typical for males. Then followed psychotic, neurotic disorders and then other types of diagnoses. Depressive disorder has not been specifically defined through the database; it is dispersed in the categories of psychoses and neuroses. The suicidal method for this group was not specifically related to the suicide commitment. The lower part of the graphic presentation shows the decision trees of the group with no psychiatric diagnosis. The most common suicidal method is hanging, typical for males. This category is most frequent, following are others (shooting, jump from, been run, poisoning, gas, stab, cut and combination). In females most typical is drowning and ingestion of larger quantities of medicaments.

Females are more common in attempting suicide than males (Figure 2). Female suicide attempts are relatively uniform: the ingestion of medicaments is far more frequent than the second most common mode of a suicide attempt, this is followed by combination, poisoning, drowning and jump from heights. Males tend to use stabbing, hanging, gas, cut and shooting. It is worth mentioning that the attempted suicide by hanging ends fatally in $69 \%$ by repeated attempt (Ziherl \& Zalar 2006).

\section{DISCUSSION}

Given that suicide is a major global problem, there is a need for deeper knowledge and development of efficient prevention methods. Knowledge of the most frequently used suicide methods is crucial in the prevention strategies, since it reduces the access to lethal means of suicide (WHO 2018)

Several reviews indicate that multilevel interventions are the best choice for suicide prevention (Althaus \& Hegerl 2003, Mann et al. 2005, Rihmer et al. 2004). Such a successful example is the Nurenberg Alliance Against Depression (NAD). This multilevel approach showed a clear effect in terms of reducing suicides and suicide attempts, and it improved the prevention in a simple design (Hegerl et al. 2006).

Out of all suicidal behaviours (such as personality, individual differences, cognitive factors, social aspects, and negative life events), it is the negative life events that suicide is usually preceded by (Delgado-Gomez et al. 2012). Previous findings suggest that about $90 \%$ of people who die by suicide have a psychiatric disorder 


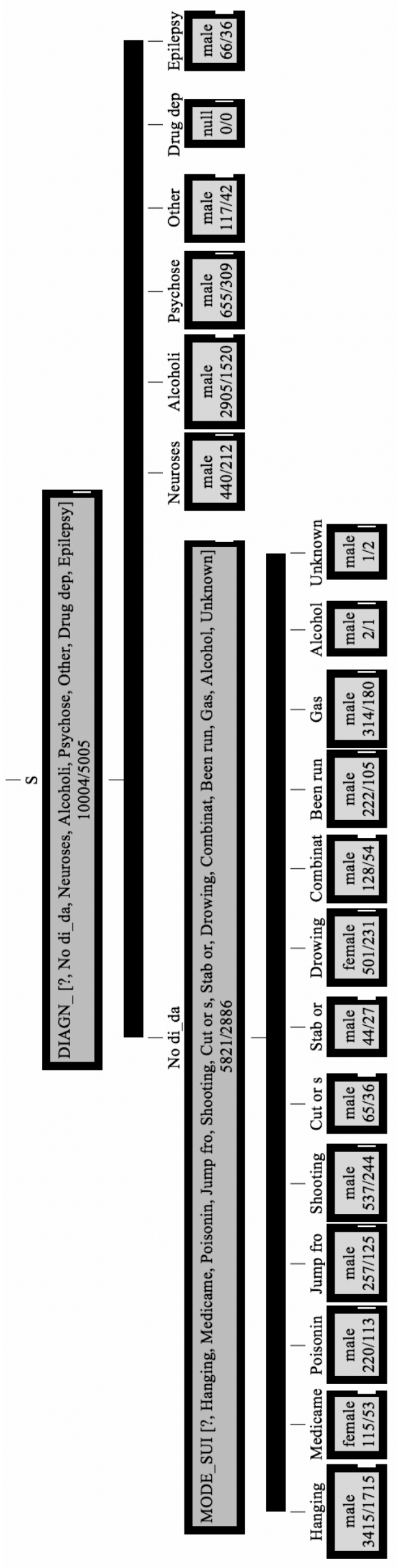

Figure 1. Suicide Commitment: method /mode and gender

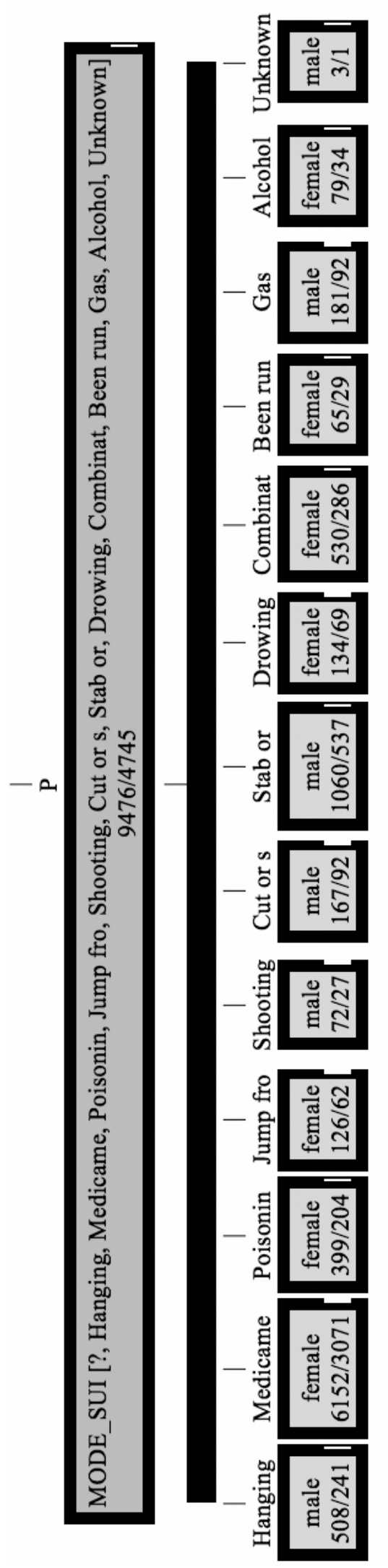

Figure 2. Suicide Attempt: method /mode and gender 
before their death, of which the majority are depressive disorders (Mann et al. 2005, Yoshimasu et al. 2008). Our results did not confirm actual presence of psychiatric diagnoses in such a high percentage. This means that improved recognition and understanding of clinical, psychological, sociological, and biological factors could help detect the high-risk individuals and select the proper treatment (Brent \& Turecki 2016). One study showed that when to the usual treatment in the program to reduce suicide rates, a brief intervention and contact was incorporated, the cases of death by suicide reduced significantly (Fleischmann et al. 2008). A significant improvement in suicide reduction was observed in the last 20 years among women, possibly because they are more likely to seek help for depression and, therefore benefit from more effective therapies (Rihmer \& Akiskal 2006). Our results contribute to suicide research

If people are informed about the frequent methods of suicide and their links with the probability of fatal consequences, they are more likely to detect unusually prepared rope, pipe or other tools for the purpose. In addition, they are more likely to hear the presence of wording revealing the plans and methods.

\section{CONCLUSION}

For studying suicide attempts, multivariate techniques proved to be useful by utilising a combination of life events, sociocultural and personality criteria (Delgado-Gomez et al. 2012). The increase of computer power enabled a wide use of data mining techniques. These methods have been used for various database screens such as WHO database or big national databases (Oquendo et al. 2012). Lately the use of data mining techniques has also became popular in psychiatry including for applications in suicidal behaviours analysis. We have used the very complex Multimethod approach, including various algorithms and approaches of Machine learning and provided additional insight into the problem of suicide in Slovenia, probably also in the mid Europe. Our attention can be directed to single methods of action, since they are broadly applicable in communication and prevention of suicide.

\section{Acknowledgements: None.}

\section{Conflict of interest: None to declare.}

\section{Contribution of individual authors:}

Bojan Zalar: concept and design of the article, review manuscript;

Blanka Kores Plesničar: review manuscript; Ina Zalar: design, writing part of manuscript, prepairing data for analysis;

Matej Mertik: multimethod analysis, writing manuscript, review manuscript.

\section{References}

1. Ajdacic-Gross $V$, Weiss $M G$, Ring $M$, Hepp U, Bopp $M$, Gutzwiller F, Rössler W: Methods of suicide: international suicide patterns derived from the WHO mortality database. Bull World Health Organ 2008; 86:726-32

2. Althaus D, Hegerl U: The evaluation of suicide prevention activities: State of the art. World J Biol Psychiatry 2003; 4:156-165

3. Bishop CM. Pattern Recognition and Machine Learning: Pattern Recognition Vol 4; 2006

4. Cibis A, Mergl R, Bramesfeld A, Althaus D, Niklewski G, Schmidtke A, et al.: Preference of lethal methods is not the only cause for higher suicide rates in males. J Affect Disord 2012; 136:9-16

5. De Ville B: Decision trees. Wiley Interdisciplinary Reviews: Computational Statistics 2013; 5:448-455

6. Delgado-Gomez D, Blasco-Fontecilla H, Sukno F, Socorro Ramos-Plasencia M, Baca-Garcia E: Suicide attempters classification: Toward predictive models of suicidal behavior. Neurocomputing 2012; 92:3-8

7. Fleischmann A, Bertolote JM, Wasserman D, De Leo D, Bolhari J, Botega NJ, et al.: Effectiveness of brief intervention and contact for suicide attempters: A randomized controlled trial in five countries. Bull World Health Organ 2008; 86:703-709

8. Ganguly S, Bhattacharjee D \& Nasipuri M: Hybrid Soft Computing Approaches: Bhattacharyya S, Dutta P, \& Chakraborty S, Eds.: Studies in Computational Intelligence 611:387-403. Springer India, 2016

9. Gupta, N: Artificial Neural Network. Network and Complex Systems 2013; 3:24-28

10. Hawton K\& Heeringen K: Suicide, The Lancet; 2009

11. Hawton K, Saunders KE, O'Connor RC: Self-harm and suicide in adolescents. Lancet 2012; 379:2373-2382

12. Hawton K, van Heeringen K: Suicide. Lancet 2009; 373:1372-1381

13. Jiang L, Li C \& Cai Z: Learning decision tree for ranking. Knowledge and Information Systems 2009; 20:123-135

14. Lester D: Changes in the methods used for suicide in 16 countries from 1960 to 1980. Acta Psychiatr Scand 1990; 81:260-1

15. Mann JJ, Apter A, Bertolote J, Beautrais A, Currier D, Haas A, et al.: Suicide prevention strategies: A systematic review. JAMA 2005; 294:2064-2074

16. McIntosh J: Methods of suicide. Maris R, Berman A, Maltsberger J, et al.: Assessment and prediction of suicide. New York, London: The Guilford Press, 1992; 382-97

17. Milčinski L: Izbrana dela. Univerzitetna psihiatrična klinika Ljubljana, 2016

18. Nock M, Borges G, Bromet E, Cha C, Kessler R \& Lee S: Suicide and suicidal behavior. Epidemiol Rev 2008; 30:133-154

19. Nock MK, Borges G, Bromet EJ, Alonso J, Angermeyer M, Beautrais $A$, et al.: Cross-national prevalence and risk factors for suicidal ideation, plans and attempts. $\mathrm{Br} J$ Psychiatry 2008; 192:98-105

20. O'Connor RC \& Nock MK: The psychology of suicidal behavior. Lancet Psychiatry 2014; 1:73-85

21. Rihmer Z \& Akiskal H: Do antidepressants t(h)reat(en) depressives? Toward a clinically judicious formulation of the antidepressant-suicidality FDA advisory in light of declining national suicide statistics from many countries. $J$ Affect Disord 2006; 94:3-13 
22. Rihmer Z, Kantor Z, Rihmer A \& Seregi K: Suicide prevention strategies $-A$ brief review. Neuropsychopharmacol Hung 2004; 6:195-199

23. Sivanandam SN \& Deepa SN: Introduction to genetic algorithms. Introduction to Genetic Algorithms. Springer Berlin Heidelberg, 2008

24. Värnik A, Kõlves K, van der Feltz-Cornelis CM, Marusic A, Oskarsson H, Palmer A, et al.: Suicide methods in Europe: a gender-specific analysis of countries participating in the "European Alliance Against Depression". J Epidemiol Community Health 2008; 62:545-51

25. WHO, Fact sheets, Suicide, 2018
26. WHO, Mental health: suicide prevention. 2014. http://www.who.int/mental_health/suicide-prevention/en/ accessed Nov 5, 2014

27. WHO, Preventing suicide: a resource for media professionals, update 2017

28. WHO, The first WHO report on suicide prevention; 2014

29. Yoshimasu K, Kiyohara $C \&$ Miyashita K: Suicidal risk factors and completedsuicide: meta-analyses based on psychological autopsy studies. Environ. Health Prev Med 2008; 13:243-256

30. Ziherl $S$ \& Zalar B: Risk of suicide after attempted suicide in the population of Slovenia from 1970 to 1996. European Psychiatry 2006; 21:396-400

\section{Correspondence:}

Prof. Bojan Zalar, MD, PhD

University Psychiatric Clinic Ljubljana

Studenec 48, 1260 Ljubljana, Slovenia

E-mail:bojan.zalar@guest.arnes.si 